\title{
Impact of Surgical Site Infection To Outcome In Patients With Operatively Treated Tibial Plateau Fractures-A Retrospective Multicenter Study
}

\section{Ralf Henkelmann ( $\sim$ ralf.henkelmann@medizin.uni-leipzig.de )}

University of Leipzig, Clinic of Orthopedic, Trauma and Plastic Surgery https://orcid.org/0000-0001-52741896

\section{Karl-Heinz Frosch}

University Hospital Hamburg-Eppendorf: Universitatsklinikum Hamburg-Eppendorf

\section{Richard Glaab}

Canton Hospital: Kantonsspital Aarau AG

\section{Meinhard Mende}

University of Leipzig Faculty of Medicine: Universitat Leipzig Medizinische Fakultat

\section{Christopher UII}

Berufsgenossenschaftliche Kliniken Bergmannsheil: Berufsgenossenschaftliches Universitatsklinikum

Bergmannsheil

\section{Philipp-Johannes Braun}

Unfallkrankenhaus Berlin

\section{Tobias J. Gensior}

Orthopädische Gemeinschaftspraxis Neuss

\section{Christoph Katthagen}

University Hospital Munster: Universitatsklinikum Munster

\section{Pierre Hepp}

University Hospital Leipzig: Universitatsklinikum Leipzig

\section{Research article}

Keywords: Outcome tibial plateau fracture, surgical site infection, KOOS, Lysholm

Posted Date: January 27th, 2021

DOI: https://doi.org/10.21203/rs.3.rs-154378/v1

License: (9) (1) This work is licensed under a Creative Commons Attribution 4.0 International License. Read Full License 


\section{Abstract}

Background: Tibial plateau fractures (TPF) can be a life changing injury. Surgical site infection (SSI) occur in 3$10 \%$ and is a feared complication. Aim of this study was to evalute the impact of SSI to outcome in patients with operatively treated TPF.

Methods: We conducted a retrospective multicenter study in seven participating countries. Between January 2005 and December 2014 all participating centers have followed up patients with SSI. In addition, three centers followed up patients without SSI as a reference group. Descriptive data and follow up data with patient reported outcome scores (KOOS, Lysholm) were evaluated. Statistic analyses were performed with IBM SPSS and twosided tests to the significance level of $a=0.05$.

Results: In summary, 287 patients (41 with SSI and 246 without SSI) with an average follow-up of $75.9 \pm 35.9$ months were included in this study. Patients with a SSI had a significant poorer Outcome in KOOS5 (48.7 \pm 23.2 vs. $71.5 \pm 23.5 ; p<0.001)$ and Lysholm $(51.4 \pm 24.0$ vs. $71.4 \pm 23.5 ; p<0.001)$ compared to patients without SSI. This significant difference was also evident in the KOOS subscores pain $(57.9 \pm 22,9$ vs. $75.0 \pm 22.3 ; p<$ $0.001)$, symptoms ( $54.5 \pm 28.8$ vs. $75.4 \pm 23.4 ; p<0.001), A D L(48.8 \pm 27.5$ vs. $80.5 \pm 22.6 ; p<0.001)$ and $Q O L$ $(37.8 \pm 31.5$ vs. $56.4 \pm 30.2 ; p=0.001)$.

Conclusion: Patients with SSI differed significantly from patients without SSI in terms of gender, smoking and drug addiction. The trauma-associated data showed a significant difference in the severity of fracture morphology, concomitant injuries (especially open fracture and compartment syndrome), and the incidence of polytraumata. In terms of PRO, a significantly poorer outcome was recorded in patients with SSI.

\section{Background}

The goal of the surgical treatment of tibial plateau fractures (TPF) is to achieve the best level of mobility and quality of life postoperatively. However, the postoperative outcome depends on many factors. The initial surgical treatment strategy is determined by the fracture morphology, soft tissue damage, concomitant injuries and the general condition of the patient. In the early postoperative phase surgical site infection (SSI) is the most feared complication. Average SSI rates of 4.5\% and more were described. [1] [2] [3] In a systematic review it was shown that only $44 \%$ have a satisfactory outcome after a deep SSI. [4] Focus of the most studies is to evaluate the generell outcome of tibial plateau fractures. [5-8]

The aim of the here presented study was to evalute the impact of SSI on the outcome after operatively treated TPF measured with patient reported outcome scores.

\section{Methods}

\section{Study design}

We performed a retrospective multicenter study at seven level I trauma centers in Germany and Switzerland. The study was approved by the leading ethical committee of the XX and all corresponding ethic committees of the participating centers. 


\section{Setting / Study size}

All patients who had surgical treated tibial plateau fracture in one of the hospitals from January 2005 through December 2014 were identified by querying the hospitals database with the International Classification of Disease (ICD) code for proximal tibia fractures. To avoid an inclusion of patients which were improperly coded, primarily operated in another hospital or were not in accordance with our inclusion and exclusion criteria charts were screened manually of every patient. All variables to be recorded were specified in advance and communicated to all participating centres in a pre-prepared spreadsheet.

\section{Participants}

Inclusion criteria are: aged $>18$, primarily treated in one of the hospitals, proximal tibia fracture according to AO/OTA 41 B or C. [9]

Exclusion criteria are: previous surgery at fracture site in another hospital, AO $41 \mathrm{~A}$ fractures, pathological fractures

\section{Variables / Data sources / measurements}

In addition to the standard parameters (age, sex, etc.), comorbidities were categorized into 4 groups according to the number of comorbidities: none, 1-3 comorbidities, $4-5$ comorbidities, $\geq 6$ comorbidities. The variables diabetes mellitus, nicotine abuse, alcohol/drug abuse as well as immunosuppressive drugs were listed separately at nominal scale level.

Accompanying injuries to the affected knee joint were classified as none, not relevant (abrasions, soft tissue injuries grade 1 according to Gustillo and Anderson) and relevant (further fractures of the affected extremity, compartment syndrome). Other concomitant injuries were categorized as none, not relevant (hematoma, abrasions, craniocerebral trauma grade 1) and relevant (fracture to other body region, craniocerebral trauma $>1$ grade). Furthermore, patients with an Injury Severity Score (ISS) $>16$ were classified as polytrauma. [10]

The fracture morphology was classified according to the AO/OTA classification. [9] Furthermore, the variables open fracture and compartment syndrome were considered.

SSI was recorded according to the definition proposed by the current protocol of the National Healthcare Safety Network, Centers for Disease Control and Prevention (CDC). These definitions are used in the German guideline of the Robert Koch-Institut (RKI) as well as by the WHO [11].

Outcome was measured with two different patient reported outcome (PRO) scores, KOOS5 (Knee injury and Osteoarthritis Outcome Score) with it's corresponding subscores (Symptoms, Pain, Function in daily living [ADL], Function/sports and recreational activities [Sport/Rec], Quality of Life [QoL]) and Lysholm score. [12-14] For the KOOS5 score (maximum 100), a lower score represented more symptoms or pain, greater difficulty performing ADLs and Sport/Rec, and poorer QoL. This rating also is valid for the Lysholm score (maximum 100).

A minimum follow-up was determined at 12 months postoperatively. A follow-up examination of the patient group with a SSI was performed in all participating centers. A reference group with patients without SSI was conducted by the additional follow-up examination of patients in three centers. 


\section{Bias}

Due to the retrospective study design and the large number of study centers, it is possible that patients with an SSI may not have returned to one of the study centers for further treatment. Thus, the number of SSIs may be higher and the influence of a higher number of patients with SSIs may have an impact on the results.

All patients with SSIs were contacted and followed up by telephone or post. Patients without SSI were included in three centers on the same procedure.

\section{Statistical methods}

The study cohort was characterized by standard statistics: Mean value (standard deviation) for continuous data, number (percent) for categorical data. Patient groups with and without infection were compared using ttest for continuous measurements and chi-square tests without correction for cross tables. All tests were performed two-sided to the significance level $a=0.05$. The analyses were performed with IBM SPSS Statistics version 24 software.

\section{Results}

\section{Participants}

Between January 2005 and Deceber 2014, 2106 patients were included in the overall study with 94 patients with SSI, which corresponds to a rate of $4.5 \%$.

Of those 94 patients, 41 could be evaluated according to the study protocol (43.6\%). As a reference group, 246 patients without SSI with follow-up were included in three centers (37.0\%). In summary, 287 patients with an average follow-up of $75.9 \pm 35.9$ months (14-146) were included in this study (Fig. 1).

\section{Descriptive Data}

Basic demographic data for the collective and the two subgroups (SSI, without SSI) are presented in Tables 1 and 2. Patients in the overall group were on average 50.7 years old, had a BMI of 26.8 and a ratio of women of $49.1 \%$. There was no significant difference between the groups in terms of age, Diabetes mellitus, BMI and comorbidities. Furthermore, there was a significant difference in body weight, sex, smoking and drug abuse between the groups (Table 1). 
Table 1

descripive data of the overall population and the subgroups with and without SSI.

\begin{tabular}{|c|c|c|c|c|c|c|c|c|}
\hline & & \multicolumn{2}{|c|}{$\operatorname{SSI}(n=41)$} & \multicolumn{2}{|c|}{ without SSI $(n=246)$} & \multicolumn{2}{|c|}{ Total $(n=287)$} & \multirow[t]{2}{*}{$p$} \\
\hline & & Mean & SD & Mean & SD & Mean & SD & \\
\hline Age & Years & 52,1 & 11,1 & 50,5 & 14,2 & 50,7 & 13,8 & 0,4 \\
\hline Weight & $\mathrm{kg}$ & 85,0 & 18,2 & 78,1 & 18,4 & 79,2 & 18,5 & 0,03 \\
\hline \multirow[t]{2}{*}{ BMI } & $\mathrm{kg} / \mathrm{m}^{2}$ & 27,7 & 5,1 & 26,7 & 5,4 & 26,8 & 5,4 & 0,2 \\
\hline & & $\mathrm{n}$ & $\%$ & $\mathrm{n}$ & $\%$ & $\mathrm{n}$ & $\%$ & \\
\hline \multirow[t]{2}{*}{ Sex } & $\mathrm{m}$ & 28 & $68,3 \%$ & 113 & $45,9 \%$ & 141 & $49,1 \%$ & \multirow[t]{2}{*}{0,01} \\
\hline & $f$ & 13 & $31,7 \%$ & 133 & $54,1 \%$ & 146 & $50,9 \%$ & \\
\hline \multirow[t]{4}{*}{ Comorbidities } & none & 20 & $48,8 \%$ & 136 & $55,3 \%$ & 156 & $54,4 \%$ & \\
\hline & $1-3$ & 17 & $41,5 \%$ & 95 & $38,6 \%$ & 112 & $39,0 \%$ & \\
\hline & $4-5$ & 3 & $7,3 \%$ & 4 & $1,6 \%$ & 7 & $2,4 \%$ & \\
\hline & $\geq 6$ & 1 & $2,4 \%$ & 7 & $2,9 \%$ & 8 & $2,8 \%$ & 0,2 \\
\hline Diabetes mellitus & & 5 & $12,2 \%$ & 16 & $6,6 \%$ & 21 & $7,3 \%$ & 0,3 \\
\hline Immunosuppression & & 1 & $2,4 \%$ & 3 & $1,2 \%$ & 4 & $1,4 \%$ & \\
\hline Smoking & & 16 & $39,0 \%$ & 50 & $20,3 \%$ & 66 & $23,0 \%$ & 0,03 \\
\hline Drug abuse & & 7 & $17,1 \%$ & 12 & $4,9 \%$ & 19 & $6,6 \%$ & 0,01 \\
\hline
\end{tabular}

There was a significantly higher proportion of $\mathrm{C}$ fractures in the SSI group. Also in the subgroups, the SSI group showed a significantly more complex fracture morphology. Significantly more patients were polytraumatized or had a relevant concomitant injury to the affected knee joint or other body region. Open fractures or compartment syndrome were significantly more frequent in the SSI group (Table 2). 
Table 2

data of the overall population and the subgroups with and without SSI concerning fracture morphology and concomitant injuries

\begin{tabular}{|c|c|c|c|c|c|c|c|c|}
\hline & & \multicolumn{2}{|c|}{ SSI $(n=41)$} & \multicolumn{2}{|c|}{$\begin{array}{l}\text { without SSI }(n= \\
246)\end{array}$} & \multicolumn{2}{|c|}{$\begin{array}{l}\text { Total }(n= \\
287)\end{array}$} & \multirow[t]{2}{*}{$p$} \\
\hline & & $\mathbf{n}$ & $\%$ & $\mathbf{n}$ & $\%$ & $\mathrm{n}$ & $\%$ & \\
\hline \multirow[t]{2}{*}{ AO categories } & $B$ & 8 & $19,5 \%$ & 142 & $57,7 \%$ & 150 & $52,3 \%$ & \multirow{2}{*}{$\begin{array}{l}< \\
0,001\end{array}$} \\
\hline & C & 33 & $80,5 \%$ & 104 & $42,3 \%$ & 137 & $47,7 \%$ & \\
\hline \multicolumn{9}{|l|}{ AO subcategories } \\
\hline & B1 & 2 & $4,8 \%$ & 24 & $9,8 \%$ & 26 & $9,1 \%$ & \\
\hline & B2 & 1 & $2,4 \%$ & 44 & $18,0 \%$ & 45 & $15,7 \%$ & \\
\hline & B3 & 5 & $12,2 \%$ & 74 & $30,3 \%$ & 79 & $27,5 \%$ & \\
\hline & C1 & 3 & $7,3 \%$ & 18 & $7,4 \%$ & 21 & $7,3 \%$ & \\
\hline & $\mathrm{C} 2$ & 6 & $14,6 \%$ & 12 & $4,9 \%$ & 18 & $6,3 \%$ & \\
\hline & C3 & 24 & $58,5 \%$ & 74 & $30,3 \%$ & 98 & $34,1 \%$ & \\
\hline concomitant injury of affected knee & & 22 & $53,6 \%$ & 96 & $36,4 \%$ & 118 & $41,1 \%$ & 0,01 \\
\hline compartment syndrome & & 16 & $39,0 \%$ & 14 & $5,7 \%$ & 30 & $10,5 \%$ & $\begin{array}{l}<, 001 \\
0,01\end{array}$ \\
\hline open fracture & & 9 & $22,0 \%$ & 7 & $2,9 \%$ & 16 & $5,6 \%$ & $\begin{array}{l}<, 001 \\
0,00\end{array}$ \\
\hline $\begin{array}{l}\text { concomitant injury of other body } \\
\text { region }\end{array}$ & & 20 & $48,8 \%$ & 47 & $19,3 \%$ & 67 & $23,3 \%$ & $\begin{array}{l}<, 001 \\
0,0\end{array}$ \\
\hline polytrauma & & 8 & $19,5 \%$ & 17 & $7,0 \%$ & 25 & $8,7 \%$ & 0,01 \\
\hline
\end{tabular}

In surgical care, an external fixator was applied significantly more frequently in patients with an SSI. A list of the primary surgical procedures in both groups and in the overall group is shown in Table 3. 
Table 3

surgical procedures of the whole group and the subgroups with and without SSI

\begin{tabular}{|lllllll|}
\hline surgical procedure & \multicolumn{2}{c}{ SSI $(\mathbf{n = 4 1})$} & \multicolumn{2}{c}{ without SSI $(\mathbf{n = 2 4 6 )}$} & \multicolumn{2}{c|}{ Total $(\mathbf{n = 2 8 7})$} \\
\hline external fixation & $\mathbf{n}$ & $\%$ & $\mathbf{n}$ & $\%$ & $\mathbf{n}$ & $\%$ \\
\hline plate & 26 & $63,4 \%$ & 55 & $22,6 \%$ & 81 & $28,2 \%$ \\
\hline screw & 8 & $19,5 \%$ & 69 & $28,3 \%$ & 77 & $26,8 \%$ \\
\hline plate and screw & 3 & $7,2 \%$ & 75 & $30,8 \%$ & 78 & $27,2 \%$ \\
\hline double plate & 3 & $7,2 \%$ & 14 & $5,7 \%$ & 17 & $5,9 \%$ \\
\hline TKA & 0 & $0,0 \%$ & 2 & $0,8 \%$ & 2 & $0,7 \%$ \\
\hline Other & 0 & $0,0 \%$ & 3 & $1,2 \%$ & 3 & $1,0 \%$ \\
\hline
\end{tabular}

TKA; total knee arthroplasty, SSI; surgical site infection

\section{Ouctome Data}

Patients with a SSI had a significant poorer Outcome in KOOS5 (48.7 \pm 23.2 vs. $71.5 \pm 23.5)$ and Lysholm (51.4 \pm 24.0 vs. $71.4 \pm 23.5$ ) compared to patients without SSI (Fig. 2, Table 4).

This significant difference was also evident in the KOOS subscores pain ( $57.9 \pm 22,9 \mathrm{vs} .75 .0 \pm 22.3)$, symptoms (54.5 \pm 28.8 vs. $75.4 \pm 23.4)$, ADL (48.8 \pm 27.5 vs. $80.5 \pm 22.6)$ and QOL (37.8 \pm 31.5 vs. $56.4 \pm 30.2)$. In the subscore Sports/Rec (36.9 \pm 37.1 vs. $41.0 \pm 35.7)$ there was no significant difference between the groups (Fig. 2, Table 4). Furthermore the Lysholm score differed significantly between the groups $(51.4 \pm 24.0$ vs. $71.4 \pm 23.5$, $p$ $=0.001)$ 
Table 4

Outcome by KOOS5 and Subscores and Lysholm for whole group and subgroub with and without SSI

\begin{tabular}{|c|c|c|c|c|c|c|c|c|c|c|c|c|}
\hline & \multicolumn{4}{|c|}{ SSI $(n=41)$} & \multicolumn{8}{|c|}{ without SSI $(n=246)$} \\
\hline & Mean & SD & Min & $\operatorname{Max}$ & Mean & SD & Min & $\operatorname{Max}$ & diff & \multicolumn{2}{|c|}{$\begin{array}{l}95 \% \\
\text { confidence } \\
\text { interval }\end{array}$} & $\begin{array}{l}\mathrm{P} \\
\text { value }\end{array}$ \\
\hline $\begin{array}{l}\text { KOOS } \\
\text { sympt }\end{array}$ & 57,9 & 22,9 & 14,3 & 100 & 75,0 & 22,3 & 10,7 & 100 & $-17,1$ & $-25,0$ & $-9,3$ & $\begin{array}{l}< \\
0.001\end{array}$ \\
\hline $\begin{array}{l}\text { KOOS } \\
\text { pain }\end{array}$ & 54,5 & 28,8 & 0,0 & 100 & 75,4 & 23,4 & 2,8 & 100 & $-20,9$ & $-30,5$ & $-11,2$ & $\begin{array}{l}< \\
0.001\end{array}$ \\
\hline $\begin{array}{l}\text { KOOS } \\
\text { ADL }\end{array}$ & 48,8 & 27,5 & 0,0 & 100 & 80,5 & 22,6 & 0,0 & 100 & $-31,7$ & $-40,9$ & $-22,5$ & $\begin{array}{l}< \\
0.001\end{array}$ \\
\hline $\begin{array}{l}\text { KOOS } \\
\text { Sport/Rec }\end{array}$ & 36,9 & 37,1 & 0,0 & 100 & 41,0 & 35,7 & 0,0 & 100 & $-4,2$ & $-16,9$ & 8,5 & 0,51 \\
\hline $\begin{array}{l}\text { KOOS } \\
\text { QoL }\end{array}$ & 37,8 & 31,5 & 0,0 & 100 & 56,4 & 30,2 & 0,00 & 100 & $-18,7$ & $-29,4$ & $-7,9$ & 0.001 \\
\hline KOOS 5 & 48,7 & 23,2 & 5,4 & 97,6 & 71,5 & 21,8 & 7,00 & 100 & $-22,8$ & $-30,7$ & $-14,9$ & $\begin{array}{l}< \\
0.001\end{array}$ \\
\hline
\end{tabular}

SD; standard deviation; ADL, Function in daily living; Sport/Rec, function /sports and recreational activities; QoL, Quality of Life

\section{Discussion}

The aim of this study was to evaluate the outcome of patients with operatively treated TPF with SSI compared to patients without SSI using established and validated PROs. In terms of PRO, a significantly poorer outcome was recorded in patients with SSI. Furthermore, our analysis showed that patients with SSI differed significantly from patients without SSI in terms of gender, smoking and drug addiction. Additionally the trauma-associated factors showed a significant difference in the severity of fracture morphology, concomitant injuries (especially open fracture and compartment syndrome), and the incidence of polytraumata between the groups. The influence of some of these factors on the development of an SSI has already been demonstrated by us hence a distribution of these factors in the groups was to be expected. [15]

In general TPF can be life-changing injuries for concerned patients. This can be additionally highlighted by our data compared to KOOS or Lysholm reference data from a healthy population. [14, 16]

However, an SSI significantly further worsens the outcome. We have already been able to show in a systematic review that an infection is associated with a poor outcome. [4] But the influence of SSI has not yet been investigated in multiple studies with PROs. Van den Berg et al. could not show any difference between patients with or without SSI in their subgroup analysis, which only had a small number of cases $(n=6)$. [6] Our results of the whole group in the KOOS subscores are comparable with the results of other studies. [5-7, 17] Finally, a significant negative influence of the SSI was found in Lysholm, KOOS5 and in four of five KOOS subscores. With regard to pain, symptoms, $A D L$ and QoL the long hospital stay with the associated changes in terms of atrophy 
and movement limitation as well as the impairment of the periarticular soft tissues by multiple revisions could mainly be responsible for this. In general a return to sport is often very difficult after TPF as also shown in other studies and may explain why there was no difference between the groups. [5-7] Also the Lysholm Score confirmed the negative influence of an infection on the outcome.

In addition to a SSI, other important factors should be considered which can cause a poor outcome. [17-20] Singleton et al. were able to show that the KOOS worsens depending on the articular congruity after reduction. [8] However, patients with an SSI had a significantly worse outcome (e.g. KOOS pain 54.5, KOOS ADL 48.8, KOOS QoL 37.8) compared to the subgroup of patients with an amount of more than $5 \mathrm{~mm}$ articular depression (e.g. KOOS pain 69.4, KOOS ADL 78.1, KOOS QoL 52.8). Furthermore Timmers et al. formed two subgroups regarding the outcome with KOOS in low- and high-energy trauma. This showed a significantly poorer result in the group with a high-energy trauma (e.g. KOOS pain $66.0 \pm 30.3$, KOOS ADL 68.1 \pm 28 .0). [5] Compared to patients with high-energy trauma, patients with SSI remained significantly poorer (e.g. KOOS pain $54.5 \pm 28.8$; KOOS ADL $48.8 \pm 27.5$ ). A further study found significant differences in the outcome with regard to the fracture morphology. [17] This was also a factor to be expected. In comparison to the study of van Dreumet et al. in our study, a patient with a SSI, regardless of his fracture morphology, has a significantly worse result than without a SSI. Jansen et al evaluated 23 AO C fractures (follow up 67 months) with an overall KOOS of 67.84 and Lysholm of 66.2 compared to our patients with a SSI (KOOS5 $48.7 \pm 23.2$, Lysholm $51.4 \pm 24.0$ ) those are significantly better. Even patients with a postoperative malalignement had a better outcome (KOOS $58 \pm 14$ ) than patients with a SSI. [21]

Finally, our data showed that an SSI is responsible for a poor outcome after surgical treatment of a TPF regardless of other risk or influencing factors. These strong results show that in addition to optimal fracture reduction and postoperative management, the avoidance of SSI has the greatest impact on a good outcome in TPF.

\section{Limitations}

The strongest limitation is the retrospective study design. Epidemiological data were complete from all participating centers, but only a small number of patients could be obtained for follow-up (43.6\% and 37.0\%). Due to this small number of responders, a bias cannot be excluded. However, in terms of epidemiological data, there was no significant difference between the groups with and without SSI in terms of with and without followup.

\section{Abbreviations}

$\mathrm{ADL}$

Activities of daily living

AO/OTA

Arbeitsgemeinschaft für Osteosynthesefragen Foundation/Orthopaedic Trauma Association classification BMI

Body mass index

$\mathrm{CDC}$

Centers for Disease Control and Prevention

FU 
follow-up

KOOS

Knee Osteoarthritis Outcome Score

ICD

International Classification of Diseases

ISS

Injury Severity Score

PRO

patient reported outcom

QoL

Quality of life

SD

Standard deviation

Sport/Rec

Sports and recreation

SSI

Surgical site infection

TKA

total knee arthroplasty

TPF

tibial plateau fracture

\section{Declarations}

Ethics approval and consent to participate

This study was performed in line with the principles of the Declaration of Helsinki. The study was approved by the leading ethical committee of the University of Leipzig (Reference number: 098/15-ff) and by the corresponding ethics committees of all participating hospitals. Patients gave their written consent to participate in the study after receiving appropriate information.

Consent for publication

Not applicable.

Availability of data and materials

The datasets used and/or analyzed during the current study are available from the corresponding author on reasonable request.

Conflict of interest

The authors declare that they have no conflict of interest

Funding 
None.

\section{Authors' contributions}

$\mathrm{RH}$ and $\mathrm{PH}$ were responsible for data control and study supervision. The first draft of the manuscript was written by $\mathrm{RH}$ and $\mathrm{PH}$; all authors commented on previous versions of the manuscript. All authors read and approved the final manuscript. RH, TG, CU, PJB, CK, RG, and KHF performed the data acquisition and data control in each trial site and obtained the respective approval of the responsible ethics committee. MM performed data curation, formal analysis, validation, and visualization of the study data, as well as further review and revision of the manuscript. $\mathrm{PH}$ and $\mathrm{RH}$ were responsible for project coordination.

\section{Acknowledgements}

We would like to thank Editage (www.editage.com) for English language editing.

\section{References}

1. Momaya AM, Hlavacek J, Etier B, Johannesmeyer D, Oladeji LO, Niemeier TE, et al. Risk factors for infection after operative fixation of Tibial plateau fractures. Injury. 2016;47:1501-5. doi:10.1016/j.injury.2016.04.011.

2. Norris GR, Checketts JX, Scott JT, Vassar M, Norris BL, Giannoudis PV. Prevalence of Deep Surgical Site Infection After Repair of Periarticular Knee Fractures: A Systematic Review and Meta-analysis. JAMA Netw Open. 2019;2:e199951. doi:10.1001/jamanetworkopen.2019.9951.

3. Ochen Y, Peek J, McTague MF, Weaver MJ, van der Velde D, Houwert RM, Heng M. Long-term outcomes after open reduction and internal fixation of bicondylar tibial plateau fractures. Injury. 2020;51:1097-102. doi:10.1016/j.injury.2020.03.003.

4. Henkelmann R, Frosch K-H, Glaab R, Lill H, Schoepp C, Seybold D, et al. Infection following fractures of the proximal tibia - a systematic review of incidence and outcome. BMC Musculoskelet Disord. 2017;18:481. doi:10.1186/s12891-017-1847-z.

5. Timmers TK, van der Ven DJC, Vries LS de, van Olden GDJ. Functional outcome after tibial plateau fracture osteosynthesis: a mean follow-up of 6 years. Knee. 2014;21:1210-5. doi:10.1016/j.knee.2014.09.011.

6. van den Berg J, Reul M, Nunes Cardozo M, Starovoyt A, Geusens E, Nijs S, Hoekstra H. Functional outcome of intra-articular tibial plateau fractures: the impact of posterior column fractures. Int Orthop. 2017;41:1865-73. doi:10.1007/s00264-017-3566-3.

7. Evangelopoulos D, Chalikias S, Michalos M, Vasilakos D, Pappa E, Zisis K, et al. Medium-Term Results after Surgical Treatment of High-Energy Tibial Plateau Fractures. J Knee Surg. 2019. doi:10.1055/s-00391677822.

8. Singleton N, Sahakian V, Muir D. Outcome After Tibial Plateau Fracture: How Important Is Restoration of Articular Congruity? J Orthop Trauma. 2017;31:158-63. doi:10.1097/BOT.0000000000000762.

9. Meinberg EG, Agel J, Roberts CS, Karam MD, Kellam JF. Fracture and Dislocation Classification Compendium-2018. J Orthop Trauma. 2018;32(Suppl 1):1-170. doi:10.1097/BOT.0000000000001063.

10. Baker SP, O'Neill B, Haddon W, Long WB. The injury severity score: a method for describing patients with multiple injuries and evaluating emergency care. J Trauma. 1974;14:187-96. 
11. CDC Definition SSI. http://www.cdc.gov/nhsn/pdfs/pscmanual/9pscssicurrent.pdf. Accessed 7 Sep 2018.

12. Kessler S, Lang S, Puhl W, Stöve J. Der Knee Injury and Osteoarthritis Outcome Score-ein Funktionsfragebogen zur Outcome-Messung in der Knieendoprothetik. Z Orthop Ihre Grenzgeb. 2003;141:277-82. doi:10.1055/s-2003-40083.

13. Roos EM, Roos HP, Lohmander LS, Ekdahl C, Beynnon BD. Knee Injury and Osteoarthritis Outcome Score (KOOS)--development of a self-administered outcome measure. J Orthop Sports Phys Ther. 1998;28:88-96. doi:10.2519/jospt.1998.28.2.88.

14. Briggs KK, Steadman JR, Hay CJ, Hines SL. Lysholm score and Tegner activity level in individuals with normal knees. Am J Sports Med. 2009;37:898-901. doi:10.1177/0363546508330149.

15. Henkelmann R, Frosch K-H, Mende M, Gensior TJ, Ull C, Braun P-J, et al. Risk factors for deep surgical site infection in patients with operatively treated tibial plateau fractures: A retrospective multicenter study. J Orthop Trauma. 2020. doi:10.1097/BOT.0000000000002011.

16. Paradowski PT, Bergman S, Sundén-Lundius A, Lohmander LS, Roos EM. Knee complaints vary with age and gender in the adult population. Population-based reference data for the Knee injury and Osteoarthritis Outcome Score (KOOS). BMC Musculoskelet Disord. 2006;7:38. doi:10.1186/1471-2474-7-38.

17. van Dreumel RLM, van Wunnik BPW, Janssen L, Simons PCG, Janzing HMJ. Mid- to long-term functional outcome after open reduction and internal fixation of tibial plateau fractures. Injury. 2015;46:1608-12. doi:10.1016/j.injury.2015.05.035.

18. Rademakers MV, Kerkhoffs GMMJ, Sierevelt IN, Raaymakers ELFB, Marti RK. Operative treatment of 109 tibial plateau fractures: five- to 27-year follow-up results. J Orthop Trauma. 2007;21:5-10. doi:10.1097/BOT.0b013e31802c5b51.

19. Bäumlein M, Hanke A, Gueorguiev B, Nerlich M, Liodakis E, Perren T, et al. Long-term outcome after surgical treatment of intra-articular tibial plateau fractures in skiers. Arch Orthop Trauma Surg. 2019. doi:10.1007/s00402-019-03150-6.

20. Reahl GB, Marinos D, O’Hara NN, Howe A, Degani Y, Wise B, et al. Risk Factors for Knee Stiffness Surgery After Tibial Plateau Fracture Fixation. J Orthop Trauma. 2018;32:e339-43. doi:10.1097/BOT.0000000000001237.

21. Jansen H, Frey SP, Doht S, Fehske K, Meffert RH. Medium-term results after complex intra-articular fractures of the tibial plateau. J Orthop Sci. 2013;18:569-77. doi:10.1007/s00776-013-0404-3.

\section{Figures}




\section{Flow Diagram}

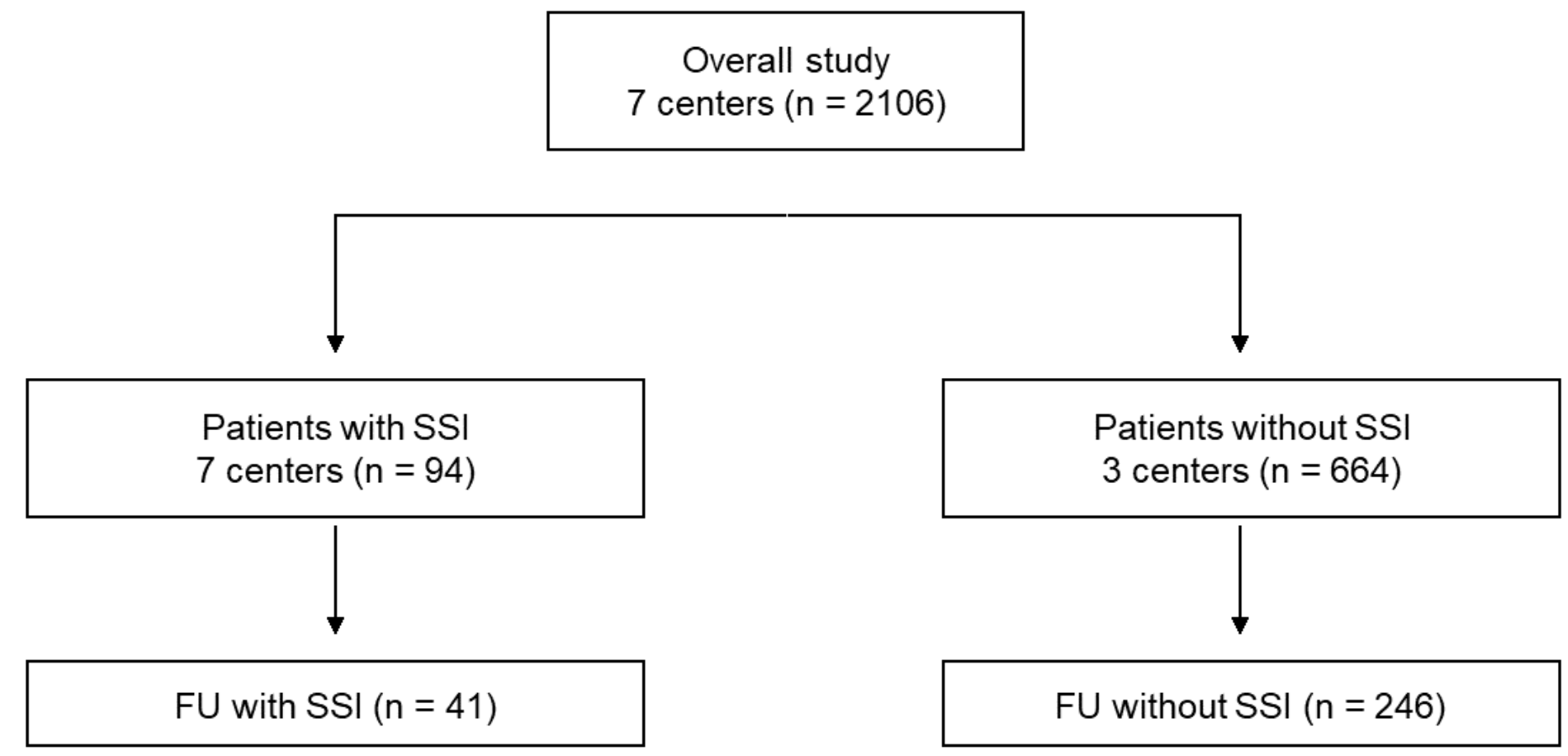

Figure 1

Flow Diagram to visualize patient inclusion from different centers. FU = follow-up, SSI = surgical site infection
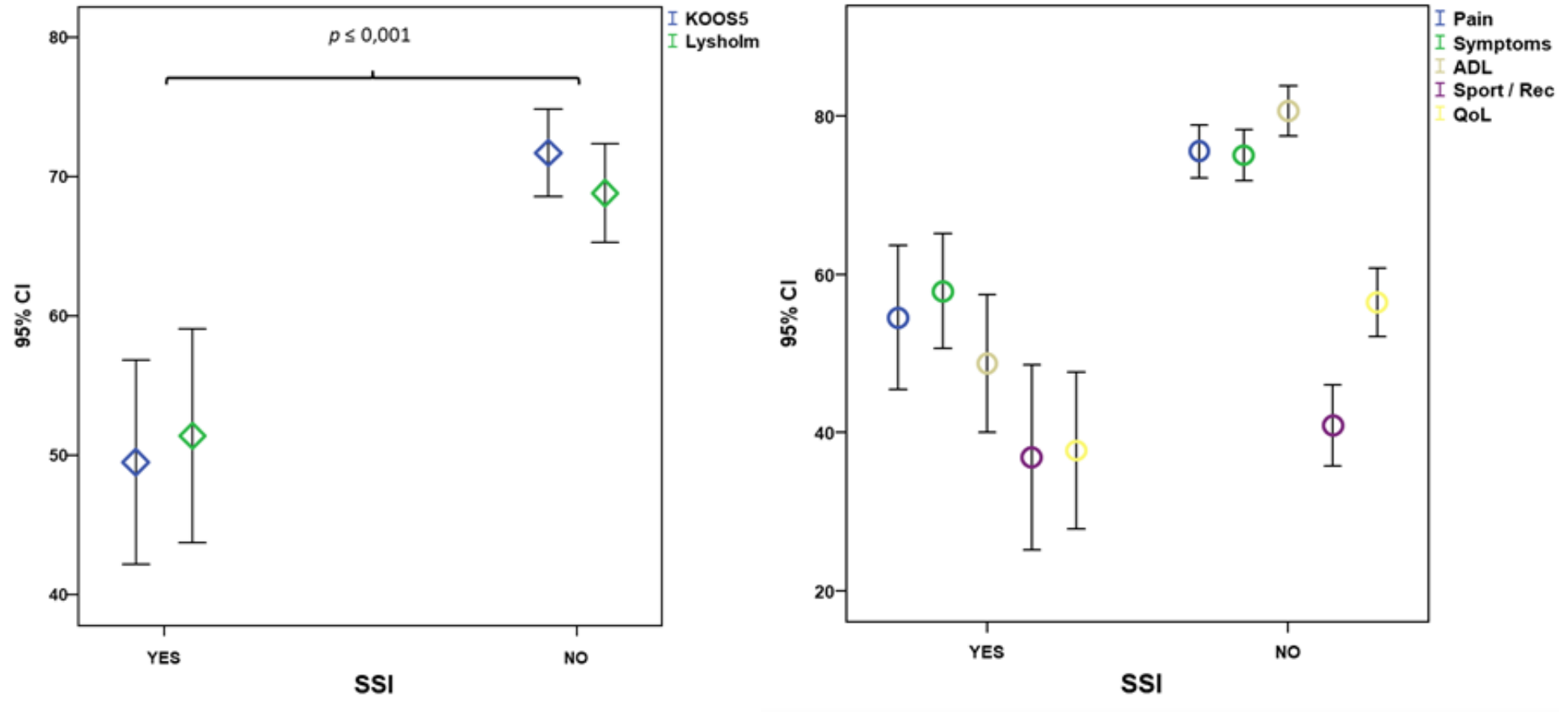

Figure 2

KOOS5 and Lysholm and KOOS Subscores compared with and without SSI (ADL, Function in daily living; Sport/Rec, function /sports and recreational activities; QoL, Quality of Life) 